\title{
Addressing Pseudo-Symmetric Misindexing in EBSD Analysis of $\gamma$-TiAl with High Accuracy Band Detection
}

\author{
Scott Sitzman ${ }^{1}$, Niels-Henrik Schmidt ${ }^{2}$, Alberto Palomares-García ${ }^{3}$, Rocío Muñoz-Moreno ${ }^{4}$, and Jenny \\ Goulden $^{2}$ \\ 1. Oxford Instruments America, Inc., Concord, MA, USA \\ 2. Oxford Instruments NanoAnalysis, High Wycombe, Buckinghamshire, UK \\ 3. IMDEA Materials Institute, Madrid, Spain \\ 4. Rolls-Royce UTC, University of Cambridge, Cambridge, UK
}

Recent technological developments in EBSD has enabled great improvements in indexing reliability and accuracy [1], including for complex, multi-phase samples and phases that traditionally present difficult indexing challenges, such as $\gamma$-TiAl. High speed cameras deliver this capability at relatively high speeds since higher quality settings, e.g. lower CCD binning, do not necessarily mean slow data collection rates. However, some individual phases continue to pose considerable indexing challenges, especially those that present extremely similar Kikuchi patterns to the EBSD camera for different crystallographic orientations, in which case the indexing engine may not clearly discern the correct orientation solution. This phenomenon is called "pseudosymmetry", as it commonly involves relatively high intensity bands in certain patterns with an apparent higher symmetry than the crystal structure actually possesses. In many cases, only very slight differences in inter-band angle separate candidate solutions, and only robust and accurate band detection may identify the correct one among them.

Conventional Hough-based band detection methods used in commercial EBSD systems since the early 1990s are sufficiently accurate for the great majority of indexing requirements. High accuracy Hough transform settings improves band detection accuracy, and is useful in mitigating pseudosymmetric misindexing [2]. However, these settings result in greater image transform time and reduced data acquisition speeds, and even modified Hough-based band detection alone may not completely eliminate pseudosymmetry problems in the most chronic cases.

New band detection refinement methods promise to improve EBSD indexing performance for some of the most chronic and important cases, and allow reasonable data acquisition speeds. These methods deliver higher accuracy band detection by iteratively comparing the positions of simulated bands with bands in the actual Kikuchi pattern image, using expected versus actual band widths and, importantly, accounting for the hyperbolic shape of bands on the phosphor screen. In addition to delivering high precision crystallographic orientation data and helping discriminate different phases with similar Kikuchi patterns, this method is sufficiently sensitive to resolve fine differences in inter-band angle to nearly eliminate many cases of pseudosymmetric misindexing. For especially difficult cases, known single-phase pseudo-symmetric orientation relationships may be specifically examined by the system to help further resolve these indexing issues.

One important application is for $\gamma$-TiAl alloys, which are very promising jet engine turbine materials, combining low density with good oxidation and creep resistance. The high temperature deformation behaviour of these alloys needs to be better understood before they can widely replace the higher density Ni-base superalloys currently used; For example, an improved knowledge of the fundamentals of 
crystallographic slip and its interaction with the $\gamma / \gamma$ lamellar variants could be critical. Microstructural characterization is key in this effort, and EBSD has an important role to play. Pseudosymmetry, however, is a major issue here (Fig. 1a), arising from $\gamma$-TiAl's close tetragonal c:a unit cell parameter ratio of 1.018, giving the Kikuchi patterns it generates a pseudo-cubic configuration, resulting in indexing inaccuracies with common $90^{\circ}$ orientation solution mistakes about the primary prismatic axes. These mistakes show the same misorientations as boundaries between real $\gamma$-TiAl lamellae, causing further problems in revealing the true microstructure. Phase discrimination between coexisting $\gamma(\mathrm{TiAl})$ and $\alpha_{2}\left(\mathrm{Ti}_{3} \mathrm{Al}\right)$ phases can also be difficult (Fig. 2a). Application of a new, automated band detection tool and system knowledge of the confronting pseudo-symmetry almost completely eliminate these issues (Fig.'s 1b and 2b) and are used in real-time during data collection, at normal acquisition speeds.

These tools may be applied for other important pseudosymmetry problems in EBSD. For example, in some solar cell materials, chalcopyrite-type phases generate Kikuchi patterns that deviate only very slightly from higher symmetry patterns, creating orientation determination problems which can undermine grain size and orientation studies if not properly addressed.

\section{References:}

[1] K. Thomsen et al., Royal Microscopy Society EBSD 2014 conference proceedings (2014)

[2] C. Zambaldi et al., J. Appl. Cryst. 42 (2009), p. 1092-1101
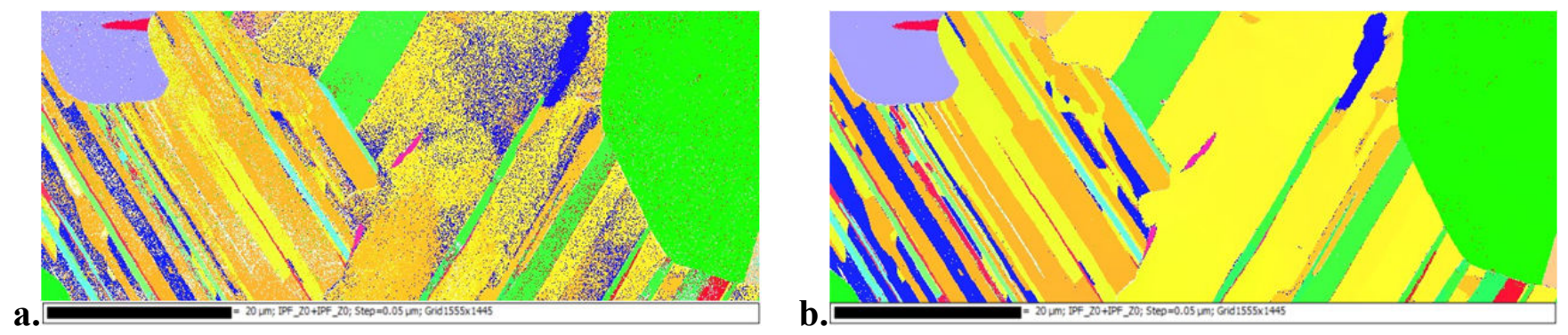

Figure 1. Comparison of results using conventional and special band detection methods for $\gamma(\mathrm{TiAl})$. (a) Orientation map from a conventional band detection dataset, exhibiting multiple orientation errors seen as speckled pixels. (b) Orientation map from a dataset using special high accuracy band detection with system knowledge of the encountered pseudosymmetry, resulting in almost no indexing errors. No postacquisition cleaning has been performed on either map. Scale bars are $20 \mu \mathrm{m}$.

a.

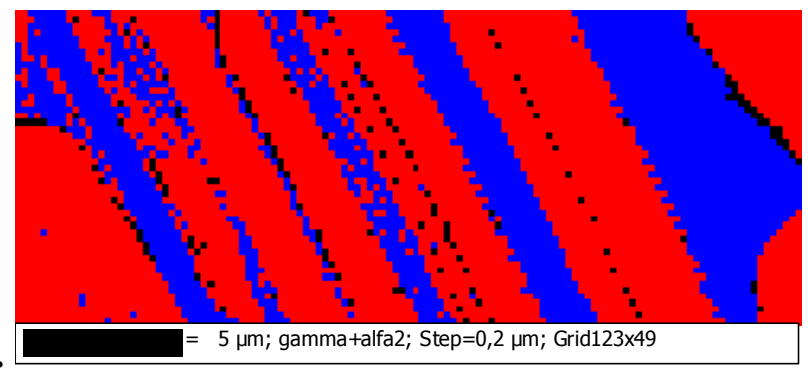

b.

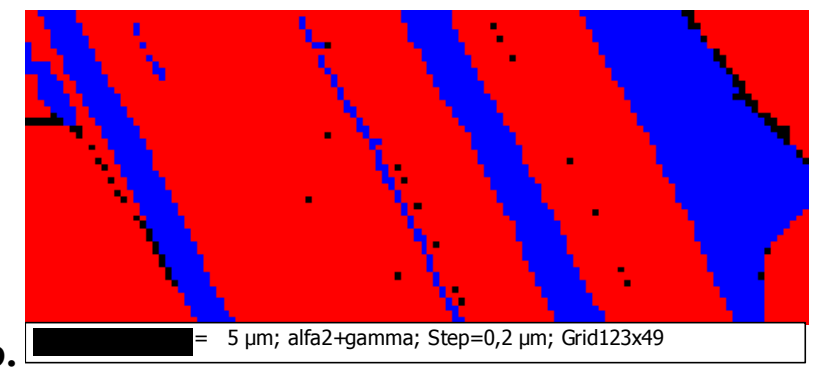

Figure 2. Comparison of band detection methods using phase maps, with red $=\gamma(\mathrm{TiAl})$, blue $=$ $\alpha_{2}\left(\mathrm{Ti}_{3} \mathrm{Al}\right)$, black $=$ no solution. (a) Conventional band detection method results in some phase discrimination mistakes, seen as individual, speckled blue and red pixels. (b) New band detection method results in nearly no indexing errors, as well as fewer non-indexed points. Maps are as-acquired, with no post-acquisition data cleaning. Scale bars are $5 \mu \mathrm{m}$. 